Original Paper http://ajol.info/index.php/ijbcs http://indexmedicus.afro.who.int

\title{
Prévalence et facteurs des risques associés au paludisme chez les patients de l'Hôpital Provincial d'Abéché (Tchad)
}

\author{
Abdelsalam Adoum DOUTOUM ${ }^{1}$, Djamalladine Mahamat DOUNGOUS ${ }^{2 *}$, \\ Elysée Gabdibé GONDIMO ${ }^{3}$, Richard Dounbé LAOUGANGTA ${ }^{3}$, Abderaman ADOUM $^{4}$, \\ Badawe GARANDI ${ }^{5}$ et Nicolas Yanou NJINTANG ${ }^{3}$
}

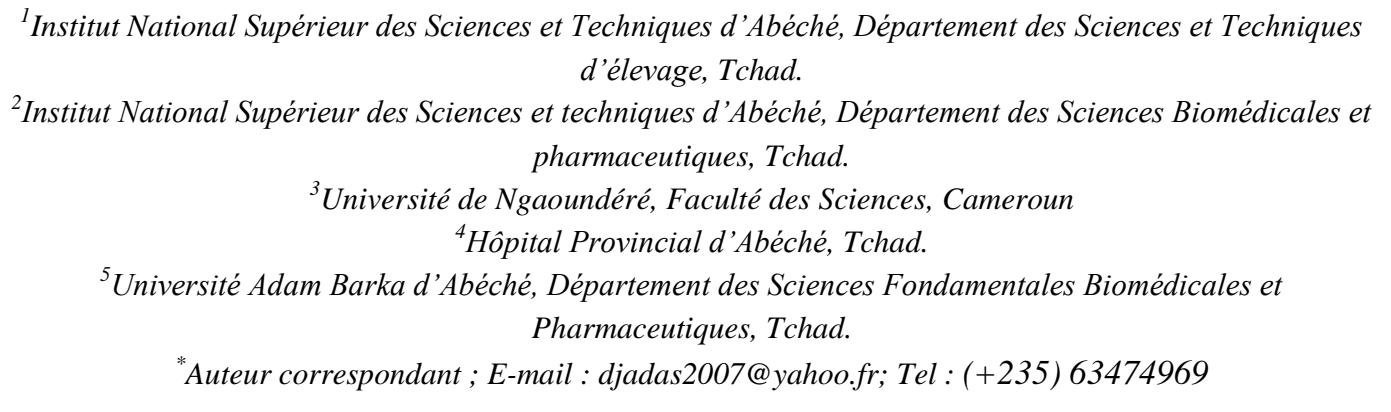

\section{RESUME}

Le paludisme est une maladie endémique en Afrique intertropicale. En vue d'évaluer les différents efforts consentis dans la lutte contre le paludisme dans le monde, l'Organisation Mondiale de la Santé (OMS) a présenté en 2016 la tendance de l'évolution de la maladie dans différents pays, grâce aux données épidémiologiques existantes. Certaines régions du Tchad et surtout Abéché n'ont pas bénéficié de cette évaluation à cause des données insuffisantes. L'objectif général de cette étude était de déterminer la prévalence du paludisme chez les patients de l'Hôpital Provincial d'Abéché (HPA) et de spécifier les facteurs des risques associés à cette pathologie endémique. Nous avons mené une étude descriptive transversale de Mai à Juin 2017 à l'HPA sur 350 patients à qui un examen de Goutte Epaisse et la Recherche d'Hématozoaire (GERH) ont été réalisés. Les patients ont été soumis à un entretien sur la base d'un questionnaire basé sur la proximité de l'eau, l'usage des moustiquaires, absence de désinsectisation. Les données collectées ont été analysées et les résultats révèlent que la prévalence du paludisme était de 36,57\% et était significativement associée à la non utilisation de moustiquaire imprégnée d'insecticide $(\mathrm{p}<0,001)$, au manque de connaissances sur le paludisme $(\mathrm{p}=0,01)$ et au fait d'habiter à proximité des eaux stagnantes $(p=0,023)$, ce qui favoriserait la forte prévalence de cette pathologie. Ces données peuvent être utilisées pour la prise de décision dans les programmes de lutte antipaludique.

(C) 2019 International Formulae Group. All rights reserved.

Mots clés: Plasmodium ; paludisme, prévalence ; maladie, facteurs de risque. 


\title{
Prevalence and risk factors associated with malaria among patients at the Abéché Provincial Hospital
}

\begin{abstract}
Malaria is an endemic disease in intertropical Africa. To evaluate the various efforts made to fight malaria in the world, the World Health Organization (WHO) presented in 2016 the trend of the evolution of the disease in different countries, thanks to epidemiological data existing. Some provinces of Chad and especially Abeche did not benefit from this assessment due to insufficient data. The general objective of this study was to determine the prevalence of malaria in patients of the Provincial Hospital of Abéché (HPA) and to specify especially the risk factors associated with this endemic pathology. We conducted a cross-sectional descriptive study from May to June 2017 at the HPA on 350 patients to whom a Thick Drop examination and Hematozoan Research (GERH) were performed. The patients were interviewed on the basis of a questionnaire based on the proximity of water, the use of mosquito nets, and no disinsection. The data collected were analyzed and the results revealed that the prevalence of malaria was $36.57 \%$ and was significantly associated with age $(\mathrm{p}=$ $0.025)$, non-use of insecticide-treated mosquito nets $(p<0.001)$, lack of knowledge about malaria $(p=0.01)$ and living near stagnant water $(\mathrm{p}=0.023)$, which would favour the high prevalence of this pathology. These data can be used for decision-making in malaria control programs.
\end{abstract}

(C) 2019 International Formulae Group. All rights reserved.

Keywords: Plasmodium, Malaria, prevalence, disease, risk factors.

\section{INTRODUCTION}

Chez l'Homme, le paludisme est une maladie parasitaire causée par cinq espèces parasitaires du genre Plasmodium que sont: Plasmodium falciparum, Plasmodium vivax, Plasmodium ovales, Plasmodium malariae et Plasmodium knowlesi (Ménard et al., 2013). Sa transmission est assurée par la piqûre de l'anophèle (Anopheles sp.) femelle (Killeen et al., 2007). Maladie cosmopolite, le paludisme a causé 438000 décès dans le monde en 2014 dont $90 \%$ incombait l'Afrique subsaharienne (OMS, 2015). En 2016, un rapport évaluant les tendances de l'évolution du paludisme dans le monde a été publié par l'OMS. Le Tchad n'a pas bénéficié de cette évaluation, à cause de données insuffisantes sur cette endémie (OMS, 2016). Cette étude avait pour but de déterminer la prévalence du paludisme chez les patients de l'Hôpital Provincial d'Abéché (HPA) et de spécifier les facteurs de risque qui lui sont associés. Il s'est agi d'une étude descriptive transversale, réalisée au service de laboratoire de l'HPA sur une période de deux mois allant de Mai à Juin 2017.

\section{MATERIEL ET METHODES \\ Cadre de l'étude}

Cette étude a été menée à l'Hôpital Provincial d'Abéché, situé dans la ville
d'Abéché, capitale de la Province du Ouaddaï, proche de la frontière soudanaise. Il est situé à l'Est du Tchad. La zone d'étude est sous l'influence du climat intertropical avec une saison sèche de 9 mois et une saison pluvieuse de 3 mois. Le régime de ces deux saisons est défini par les fluctuations entre les masses d'air sec du Nord (l'harmattan) et les masses d'air humide maritime du Sud-ouest (la mousson). La pluviométrie moyenne annuelle est d'environ $300 \mathrm{~mm}$. La température de la région est variable selon les périodes. La température moyenne annuelle à Abéché est d'environ $28^{\circ} \mathrm{C}$ avec une variation en saison froide (décembre à février), comprises entre 16 et $35^{\circ} \mathrm{C}$ et en saison sèche (avril et mai) comprises entre 25 et $41{ }^{\circ} \mathrm{C}$ (Commune d'Abéché, 2016).

\section{Echantillonnage}

La population de notre étude était constituée de 350 personnes suspectées d'atteinte par le paludisme (fièvre accompagnée ou non d'autres symptômes) sans distinction d'âge, ni de sexe. Ainsi furent inclus dans l'étude, les patients qui se sont rendus au laboratoire de l'HPA pour un examen de Goutte Epaisse/Recherche d'Hématozoaire (GERH) pendant la période de l'étude et ayant consenti à participer à l'étude. La taille de l'échantillon représentatif 
a été calculée par la formule suivante, dédiée au calcul de fréquence.

$N=\frac{\mathrm{Z}^{2} \mathrm{xP}(1-\mathrm{P})}{\mathrm{i}^{2}}$

- P: prévalence du paludisme dans la population générale ;

- Z : niveau de confiance visé ;

- I : marge d'erreur acceptable ou précision ;

- $\mathrm{N}$ : taille de l'échantillon représentatif.

En utilisant cette formule, la taille de notre échantillon $\mathrm{N}=350$ [321-353] patients.

\section{Matériel biologique}

Le matériel biologique utilisé était du sang. Un microscope optique Olympus ${ }^{\circledR}$ CX31 (Olympus, Granges, Suisse) a été utilisé pour la lecture de lames colorées au Giemsa (Cypress Diagnostics, Hulshout, Belgique).

\section{Collecte de données}

Les données ont été collectées sur la base d'un questionnaire conçu à cet effet. Les patients ont été interrogés à leur arrivée au laboratoire pour la réalisation de l'examen de GERH. Le questionnaire nous a permis de recenser les informations relatives aux caractéristiques sociodémographiques (âge, sexe, statut matrimonial, profession, religion et lieu de résidence), les connaissances qu'ont les patients vis-à-vis du paludisme (entendre parler du paludisme, connaissance de la voie de transmission) et la possession de moustiquaires imprégnées ainsi que son utilisation.

\section{Analyse des échantillons}

Une goutte de sang capillaire a été prélevée au bout du doigt de chaque patient, pour confectionner une goutte épaisse sur une lame porte-objet. Les lames confectionnées étaient ensuite séchées et colorées au Giemsa (Cypress Diagnostics, Hulshout, Belgique) dilué au $1 / 10^{\mathrm{e}}$ (5 minutes). La lecture était faite au microscope optique Olympus® CX31 (Olympus, Granges, Suisse) à l'objectif X100 avec une goutte d'huile à immersion. Lorsqu'un parasite est identifié dans le champ microscopique, le résultat est positif. Si au contraire après avoir parcouru 100 champs microscopiques, sans apercevoir de parasite, l'examen est considéré comme négatif. La lecture des lames était faite par deux microscopistes; en cas de différences de résultats, il est fait un recours à un troisième microscopistes.

\section{Analyses statistiques}

Une fiche de questionnaire a été conçue sur le logiciel Sphinx V.5. La saisie de données ainsi que leurs traitement et analyse ont été faits sur le même logiciel. La fréquence de modalités pour chaque variable a été exprimée. Le test statistique de Chi carré de pearson au seuil de significativité de 5\% ainsi que le risque relatif (ou Odds ratio selon les situations) ont été utilisés lors de l'analyse, afin de rechercher les associations entre facteurs et maladie. Le seuil de signification a été fixé pour $\mathrm{P}<0,05$.

\section{RESULTATS}

Nous avons inclus 350 patients dans notre étude. Les patients ont été interrogés et ont bénéficié d'un prélèvement sanguin. Les résultats obtenus sont répartis selon la prévalence du paludisme, les connaissances des patients sur le paludisme et les paramètres sociodémographiques.

\section{Prévalence du paludisme et facteurs de risques}

La Figure 1 présente la prévalence du paludisme dans notre échantillon. Au total 128 patients ont présenté un test positif contre 222 patients qui ont eu un test négatif, soit une prévalence du paludisme de $36,57 \%$.

\section{Sexe et âge}

Le Tableau 2 présente un croisement entre quelques facteurs sociodémographiques et la prévalence. Nous n'avons pas trouvé d'association significative entre le sexe et le risque de contracter le paludisme. Nous avons en effet observé que la fréquence de la maladie est de $35 \%$ chez les femmes et $38,6 \%$ chez les hommes. Toute fois une association significative $(p=0,025)$ a été observée entre l'âge et le risque de survenue du paludisme. La prévalence a été plus élevée chez les enfants de 0-9 ans avec 50\%, suivi des sujets de plus de 39 ans avec une prévalence de $40,3 \%$. La classe de sujets la moins touchée a été 20-29 ans avec une prévalence de 25,8\%. 


\section{Relation entre connaissances des patients sur le paludisme et risque de sa contraction}

Une association très significative a été observée entre la connaissance de la maladie et le risque de sa survenue $(p=0,01)$. En effet les patients n'ayant jamais entendu parler du paludisme avaient une prévalence plus élevée $(43,6 \%)$ comparée à $30,9 \%$ chez ceux qui ont déclaré ayant entendu parler auparavant du paludisme. De même une relation significative a été observée entre le risque de contraction du paludisme et la connaissance de sa voie de transmission $(p=0,016)$. A ce titre les patients ignorant la voie de transmission du paludisme avaient une prévalence plus élevée (42,3\%), alors que ceux qui connaissaient que la piqûre de moustique est la voie de transmission du paludisme avaient une prévalence plus basse $(25,9 \%)$. Chez les patients affirmant que la voie de transmission du paludisme est orofécale, une prévalence du paludisme de 34,9\% a été observée par cette étude. Par ailleurs il a été observé une corrélation entre les connaissances des patients sur le paludisme et le risque de sa contraction $(p=0,01)$.

\section{Relation entre paludisme et les autres paramètres enquêtés}

Le Tableau 3 résume quelques habitudes sociales en rapport avec le paludisme. Globalement passer la nuit dehors et utiliser des insecticides n'avaient pas un lien significatif avec la survenue du paludisme. En revanche, posséder une MI, dormir sous une MI et la fréquence d'utilisation de MI étaient fortement associés au risque de survenue du paludisme (Tableau 3). A cet effet la prévalence du paludisme chez les sujets possédant une MI a été de $32,8 \%$ contre $44,6 \%$ chez ceux n'en possédant pas. Mieux encore la prévalence de paludisme chez les sujets dormant sous une moustiquaire a été de $26,8 \%$ contre $50,8 \%$ chez ceux ne dormant pas sous une MI. Enfin ceux qui dorment toujours sous moustiquaires imprégnés ont une prévalence de 17,2\% alors que ceux dormant rarement sous moustiquaire ont une prévalence de 34,5\%. Une autre variable de risque de survenue du paludisme a été le fait d'habiter à proximité des eaux $(p=0,023)$. A ce titre la fréquence du paludisme chez ceux à proximité des eaux stagnantes a été de $59,1 \%$ alors que cette prévalence a été de $35,1 \%$, chez ceux qui en sont éloignés.

\section{Evaluation de connaissances sur le paludisme et utilisation de MI}

Concernant les données relatives aux connaissances des patients vis-à-vis du paludisme, $55,4 \%$ des patients avaient auparavant entendu parler du paludisme contre $44,6 \%$ qui disaient n'avoir jamais entendu parler. La majorité $(60,8 \%)$ n'avait aucune idée de la voie de transmission du paludisme et parmi eux, 8,2\% ont pensé pour leur part que le paludisme se transmet par voie oro-fécale ; seuls $30,8 \%$ connaissaient la voie de transmission. A la veille de l'interrogatoire, la majorité des patients $(68 \%)$ ont répondu avoir dormi dans la cours (à ciel ouvert) contre $32 \%$ qui avaient dormi dans une chambre. Quant à la possession de moustiquaires imprégnées (MI), $68 \%$ ont affirmé posséder de MI et seulement 51,1\% de ceux-ci ont reconnu avoir dormi sous ces MI à la veille de l'enquête. Seulement 16,6\% ont toujours dormi sous MI. En s'intéressant à la position des habitations des patients vis-à-vis des points d'eaux et ou des endroits insalubres, la grande majorité $(93,7 \%)$ n'habitait pas à proximité des points d'eau et 96,9\% ne pratiquaient pas la pulvérisation intradomiciliaire d'insecticide.

\section{Caractéristiques sociodémographiques}

Notre étude était constituée à 56,3\% des femmes avec un sex-ratio $(\mathrm{F} / \mathrm{H})$ de 1,3. La tranche d'âge la plus représentée était celle de [20-29] ans, avec une fréquence de 26,6\%. Toute fois en dehors de la tranche d'âge 10-19 ans qui a une fréquence faible, toutes les autres tranches d'âge ont une fréquence quasi identique variant de 20,3 à 26,6\%. Un taux de $61,1 \%$ était totalisé par les mariés aux dépens des célibataires. La grande majorité de notre échantillon était non instruite $(70 \%)$ et de confession religieuse musulmane $(92,9 \%$.). Le Tableau 1 présente les fréquences des caractéristiques sociodémographiques des enquêtés. 
Tableau 1 : Paramètres sociodémographiques des patients.

\begin{tabular}{|c|c|c|c|}
\hline Variables & Modalités & Effectifs (n) & Fréquence $(\%)$ \\
\hline \multicolumn{4}{|l|}{ Sexe } \\
\hline & Féminin & 197 & 56,3 \\
\hline & Masculin & 153 & 43,7 \\
\hline \multicolumn{4}{|l|}{ Age (an) } \\
\hline & $0-9$ & 74 & 21,1 \\
\hline & $10-19$ & 40 & 11,4 \\
\hline & $20-29$ & 93 & 26,6 \\
\hline & $30-39$ & 71 & 20,3 \\
\hline & $>39$ & 72 & 20,6 \\
\hline \multicolumn{4}{|l|}{ Statut matrimonial } \\
\hline & Célibataire & 136 & 38,9 \\
\hline & Marié & 214 & 61,1 \\
\hline \multicolumn{4}{|l|}{ Niveau d'instruction } \\
\hline & Primaire & 35 & 10 \\
\hline & Secondaire & 44 & 12,6 \\
\hline & Supérieur & 26 & 7,4 \\
\hline & Non instruit & 245 & 70 \\
\hline \multicolumn{4}{|l|}{ Lieu de résidence } \\
\hline & $1^{\mathrm{er}}$ arrondissement & 97 & 27,7 \\
\hline & $2^{\mathrm{e}}$ arrondissement & 13 & 3,7 \\
\hline & $3^{\mathrm{e}}$ arrondissement & 48 & 13,7 \\
\hline & $4^{\mathrm{e}}$ arrondissement & 18 & 5,1 \\
\hline & $5^{\mathrm{e}}$ arrondissement & 63 & 18 \\
\hline & $6^{\mathrm{e}}$ arrondissement & 29 & 8,3 \\
\hline & Hors de la ville & 82 & 23,4 \\
\hline \multicolumn{4}{|l|}{ Profession $(n=276)$} \\
\hline & Agriculteur /éleveur & 29 & 10,5 \\
\hline & Fonctionnaire & 12 & 4,3 \\
\hline & Ménagère & 126 & 45,7 \\
\hline & Sans emploi & 83 & 30,1 \\
\hline & Secteur informel & 26 & 9,4 \\
\hline \multicolumn{4}{|l|}{ Religion } \\
\hline & Animiste & 2 & 0,6 \\
\hline & Chrétien & 23 & 6,6 \\
\hline & Musulman & 325 & 92,9 \\
\hline Total & & 350 & 100 \\
\hline
\end{tabular}




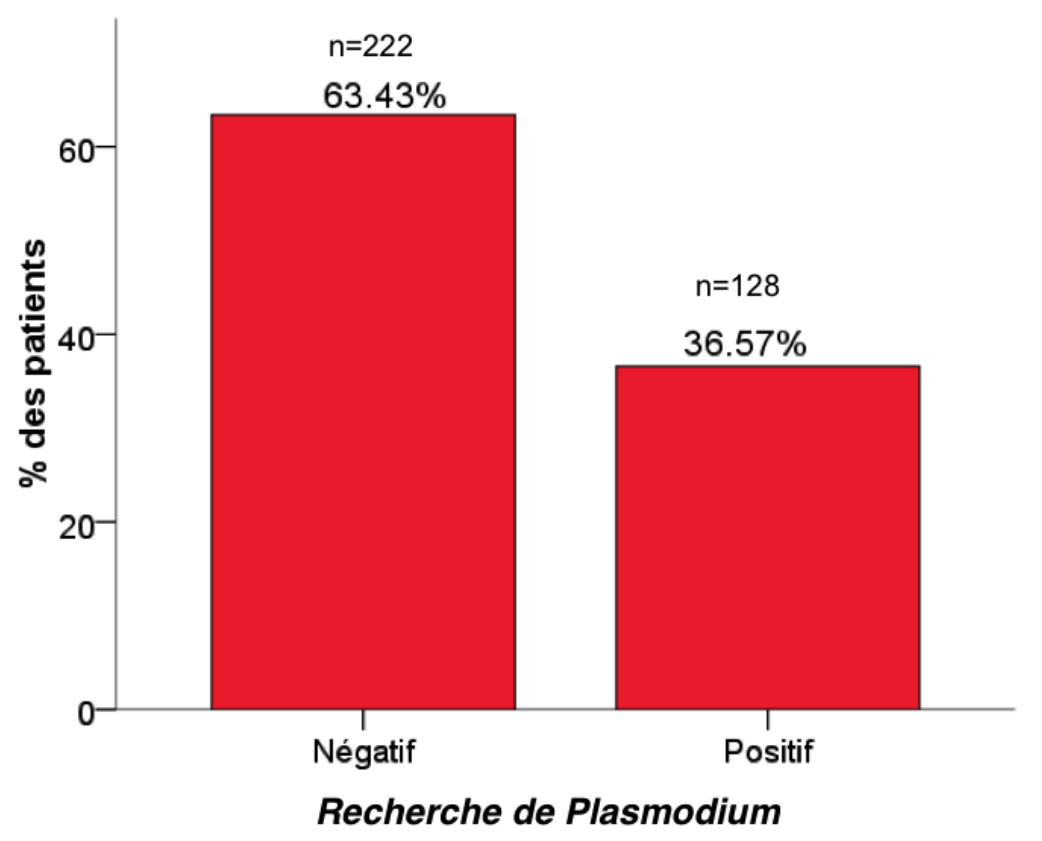

Figure 1 : prévalence du paludisme.

Tableau 2 : Distribution du paludisme en fonction du sexe et de l'âge.

\begin{tabular}{lrlll}
\hline Variables & \multicolumn{2}{l}{ Résultats de l'examen diagnostic } & P value \\
\cline { 3 - 4 } & & Négatif & Positif & \\
\hline Sexe & & & \\
& Féminin & $128(65,0 \%)$ & $69(35,0 \%)$ & \\
& Masculin & $94(61,4 \%)$ & $59(38,6 \%)$ & \\
Age (an) & & & & \\
& $0-9$ & $37(50,0 \%)$ & $37(50,0 \%)$ & \\
& $10-19$ & $27(67,5 \%)$ & $13(32,5 \%)$ & \\
$20-29$ & $69(74,2 \%)$ & $24(25,8 \%)$ & \\
$30-39$ & $46(64,8 \%)$ & $25(35,2 \%)$ & \\
$>39$ & $43(59,7 \%)$ & $29(40,3 \%)$ &
\end{tabular}


Tableau 3 : Association entre paludisme et autres paramètres enquêtés.

\begin{tabular}{|c|c|c|c|}
\hline \multirow{2}{*}{ Variables } & \multicolumn{2}{|c|}{ Résultat de l'examen diagnostic } & \multirow{2}{*}{$p$ value } \\
\hline & Négatif & Positif & \\
\hline \multicolumn{4}{|l|}{ Avoir dormi dehors } \\
\hline Non & $74(66,1 \%)$ & $38(33,9 \%)$ & $p=0,48$ \\
\hline Oui & $148(62,2 \%)$ & $90(37,8 \%)$ & \\
\hline \multicolumn{4}{|l|}{ Possession de MI } \\
\hline Non & $62(54,4 \%)$ & $50(44,6 \%)$ & $p=0,03$ \\
\hline Oui & $160(67,2 \%)$ & $78(32,8 \%)$ & \\
\hline \multicolumn{4}{|l|}{ Avoir dormi sous MI } \\
\hline Non & $29(49,2 \%)$ & $30(50,8 \%)$ & $p<0,001$ \\
\hline Oui & $131(73,2 \%)$ & $48(26,8 \%)$ & \\
\hline \multicolumn{4}{|l|}{ Fréquence d'usage de MI } \\
\hline Rare & $19(65,5 \%)$ & $10(34,5 \%)$ & \\
\hline Assez-souvent & $35(62,5 \%)$ & $21(37,1 \%)$ & $p<0,001$ \\
\hline Très souvent & $29(80,6 \%)$ & $7(19,4 \%)$ & \\
\hline Toujours & $48(82,8 \%)$ & $10(17,2 \%)$ & \\
\hline \multicolumn{4}{|l|}{ Habiter proche des eaux } \\
\hline Non & $213(64,9 \%)$ & $115(35,1 \%)$ & $p=0,023$ \\
\hline Oui & $9(40,9 \%)$ & $13(59,1 \%)$ & \\
\hline \multicolumn{4}{|l|}{ Usage d'insecticide } \\
\hline Non & $215(63,4 \%)$ & $124(36,6 \%)$ & $p=0,98$ \\
\hline Oui & $7(63,6 \%)$ & $4(36,4 \%)$ & \\
\hline
\end{tabular}

\section{DISCUSSION}

L'étude que nous avons menée a montré une prévalence du paludisme de $36,57 \%$. Ce résultat se rapproche de la prévalence observée au Mali par le Programme National de Lutte contre le Paludisme (PNLP) (2010) qui était de 37,5\%. L'estimation de la prévalence pourrait être entachée de biais. Notamment le traitement traditionnel que certains patients prendraient sans se rendre à l'hôpital pourrait induire une sous-estimation de la prévalence; ou encore le mode d'échantillonnage qui passe par une prescription de l'examen de GERH chez le médecin pourrait aussi engendrer une surestimation de cette prévalence.

La prévalence a été évaluée en rapport avec d'autres paramètres afin de déterminer si certains facteurs constitueraient des risques particuliers de survenue du paludisme. Ce faisant, une relation significative a été observée entre paludisme et l'âge $(p=0,025)$. Cette association entre âge et paludisme a été aussi observée par Brooker et al. (2009) en Tanzanie, Kenya, Ghana et Somalie. Par contre, Coulibaly et al. (2017) en Côte d'Ivoire pour leur part n'ont pas eu d'association significative entre l'âge et le paludisme. La prévalence élevée dans la tranche d'âge 0-9 ans démontre le caractère vulnérable de cette catégorie sociale. Ce résultat s'expliquerait par le fait que le système immunitaire des enfants est encore fragile et les parents prennent mois soin d'eux.

Faisant suite à l'association des facteurs à la maladie, l'ignorance des sujets impaludés était significativement liée au risque de sa contraction. Dans le même ordre de résultats, Tatoloum (2016) au Tchad, dans 
sa quête de facteurs déterminant la vulnérabilité de la population vis-à-vis du paludisme, avait incriminé le manque de connaissances comme un facteur prédisposant à la maladie. Le manque de connaissances sur le paludisme est fortement dépendant du niveau d'instruction $(p=0,01)$ et malheureusement notre échantillon était composé à $70 \%$ de personnes non sensibilisées ce qui serait une des causes majeures des prévalences élevées observées. Or, on ne peut mieux prévenir une maladie uniquement que, quand on connait sa voie de transmission et une excellente sensibilisation sur les luttes.

L'absence et la non utilisation de MI, et aussi la rareté de son utilisation sont des facteurs significativement associés au risque d'infection palustre $(p<0,001)$. En effet, on s'aperçoit qu'en faisant le rapport de la fréquence des malades parmi ceux qui dorment toujours sous MI et ceux qui n'y dorment pratiquement pas, le rapport de prévalence est de 3. Ceci étant, ceux qui dorment sans MI ont trois fois plus de chance de contracter le paludisme. Et même ceux qui $\mathrm{y}$ dorment rarement comparé à ceux qui $\mathrm{y}$ dorment toujours, le rapport est de 2. Des auteurs en Afrique ont déjà observé que dormir sans MI est un facteur à risque du paludisme à l'instar de Mukomena et al. (2016) en République Démocratique du Congo et Coulibaly et al. (2017) en Côte d'Ivoire.

Le fait d'habiter à proximité des eaux et ou des endroits insalubres (constituant probablement des gîtes larvaires) rentre dans les facteurs de vulnérabilité à l'infection palustre dans cette étude. Nos résultats corroborent ceux de Hay et al. (2000) au Kenya qui a observé que l'habitation auprès des points d'eaux est un facteur de risque du paludisme. Cette observation est similaire à celle de Kibret et al. (2015) dans leurs travaux de revue en Afrique subsaharienne, qui ont abouti à la conclusion selon laquelle, l'on a trois fois plus de chance de contracter le paludisme en habitant non loin de gîtes larvaires dans une zone de paludisme instable.
Dans notre étude, les femmes étaient plus nombreuses $(56,3 \%)$ que les hommes. Etant donné que l'examen de GERH fait partie des examens communément demandés en consultation prénatale pour protéger la mère et l'enfant, cela pourrait expliquer ce déséquilibre. La tranche d'âge la plus représentée [20-29] ans s'expliquerait pour sa part par le fait qu'à cet âge on peut décider d'aller se faire consulter pour un moindre malaise. Tandis que les plus jeunes attendent que leurs parents décident à leur place et les plus âgés vaquent à leurs occupations en essayant désespérément de supporter parfois.

En s'intéressant aux lieux de résidence de nos patients, la plupart $(27 \%)$ habitaient le premier arrondissement, suivis de ceux qui provenaient hors de la ville ou de sa périphérie $(23,4 \%)$. La localisation de l'HPA dans le premier arrondissement explique cet afflux. Ainsi, ceux qui habitent au sein de cet arrondissement profitent de cette proximité, comparativement aux patients venant d'autres arrondissements. A mesure qu'un arrondissement est éloigné de l'hôpital, l'effectif des sujets qui le représentent diminue proportionnellement et surtout si ledit arrondissement dispose d'un centre de santé.

Suivant le niveau d'instruction, les sujets étaient en majorité non scolarisés (70\%). Ce résultat confirme celui de Yandaï et al. (2017) au Tchad, qui avait une fréquence de non scolarisation de $62,4 \%$ chez les pères et $71,3 \%$ chez les mères. Pareillement, un taux de non scolarisation de 70,5\% fut observé au Tchad par le Ministère de la Santé Publique (MSP Tchad, 2013). Cette non scolarisation concerne de manière accentuée les filles $(40 \%)$.

En évaluant la connaissance des patients sur le paludisme, 55,4\% de ceux-ci avaient entendu parler auparavant du paludisme. Cette proportion s'inscrit dans la lignée de la proportion observée par Yandai et al. (2017) qui ont rapporté que $60,24 \%$ de leurs enquêtés avait entendu parler du paludisme. Cependant $60,8 \%$ de nos enquêtés n'avaient aucune connaissance sur la voie par laquelle la maladie est transmise. Cette ignorance de la voie de transmission du 
paludisme s'expliquerait par la non scolarisation élevée. En effet, en exprimant la connaissance de la voie de transmission du paludisme en fonction du niveau d'instruction, on s'aperçoit que $84,9 \%$ des non scolarisés ne connaissent pas la voie de transmission du paludisme contre seulement $18,2 \%$ de scolarisés qui ne savent pas.

A cause de la chaleur qu'il fait dans la maison pendant la saison sèche, notre étude étant faite pendant cette période, dormir hors de la maison est une habitude dans la zone sahélienne, et $68,0 \%$ de nos enquêtés ont dormi dans les cours de leurs maisons.

Quant à la possession de MI, nous avons rapporté un pourcentage de $68 \%$. Une pareille enquête fut réalisée au Mali par le PNLP (2010) ainsi que par Yandaï et al. (2017) au Tchad. Ces auteurs précités ont rapporté respectivement un taux de possession de MI de $85 \%$ et $80,6 \%$. la fréquence de possession de MI est plus basse dans notre étude, car les sujets ne sachant pas l'importance de la moustiquaire, ne connaissant pas le paludisme espéraient qu'on leur en procure (Yandaï et al., 2017).

Posséder une MI n'est pas toujours synonyme d'en faire usage car sur $68 \%$ qui possèdent une $\mathrm{MI}$, seulement $51,1 \%$ parmi eux en font usage. Au Tchad, la plupart de la population considère les MI comme un moyen d'éviter la nuisance de moustique et non celui de prévention du paludisme. L'usage a donc tendance à être relatif au degré d'attaque des moustiques. Cela pourrait d'une part justifier l'écart entre la possession et l'utilisation de MI.

\section{Conclusion}

Bien qu'évaluée pendant une période de faible transmission et dans une zone de paludisme, la prévalence de $36,57 \%$ montre que le paludisme constitue toujours un véritable problème de santé publique au Tchad et notamment à Abéché. La distribution de la maladie est étroitement liée à de nombreux facteurs qui incluent l'âge, la connaissance de la maladie, les modes de transmission et mécanismes de protection. L'éducation semble donc être le principal risque associé à cette maladie car c'est elle qui informe sur l'usage de la moustiquaire imprégnée, l'effet des eaux stagnantes. Malheureusement, au Tchad cette maladie est liée au niveau de scolarisation de la population. L'usage des langues vernaculaires et la formation des leaders sociaux pourraient également y jouer un rôle important

\section{CONFLIT D'INTERETS}

Les auteurs déclarent qu'ils n'ont aucun conflit d'intérêts.

\section{CONTRIBUTIONS DES AUTEURS}

EGG a mené l'étude de terrain et a initié le manuscrit. AAD et NYN ont initié et dirigé l'étude jusqu'à la fin. Ils ont également contribué à la correction du manuscrit. DMD a contribué à la rédaction et à la correction du manuscrit jusqu'à sa publication. RDL a contribué à la rédaction du manuscrit. AA et BG ont soutenu EGG dans les travaux de terrain.

\section{REMERCIEMENTS}

Les auteurs de cet article adressent leurs sincères remerciements aux responsables de l'Hôpital Provincial d'Abéché (HPA). Un grand merci aux patients, d'avoir accepté de participer à l'étude. Que le personnel du laboratoire de l'HPA trouve également ici l'expression de notre sincère reconnaissance.

\section{REFERENCES}

Brooker S, Kolaczinski JH, Gitonga CW, Noor AM, Snow RW. 2009. The use of schools for malaria surveillance and programme evaluation in Africa. Malaria journal, 8(1): 231.

Coulibaly G, Yao KP, Koffi M, Ahouty BA, Louhourignon LK, N'Cho M, N'Goran EK. 2017. Paludisme et parasitoses digestives chez la femme enceinte de la commune d'Abobo (Abidjan, Côte d'Ivoire). Bull. Soc. Pathol. Exot., 110(2): $85-91 . \quad$ DOI : https://doi.org/10.1007/s13149-0170552-3 
Hay SI, Omumbo JA, Craig MH, Snow RW. 2000. Earth Observation, Geographic Information Systems and Plasmodium falciparum Malaria in Sub-Saharan Africa. Adv. Parasitol., 47: 173-215.

Kibret S, Lautze J, McCartney M, Wilson GG, Nhamo L. 2015. Malaria impact of large dams in sub Saharan Africa: maps, estimates and predictions. Malar. J. 14(1): $339 . \quad$ DOI: https://doi.org/10.1186/s12936-0150873-2

Killeen GF, Smith TA, Ferguson HM, Mshinda H, Abdulla S, Lengeler C, Kachur SP. 2007.Preventingchildhood malaria in Africa by protecting adults from mosquitoes with insecticide-treated nets. PLoS Medecine, 4(7): e229. DOI : https://doi.org/10.1371/journal.pmed.004 0229

Ménard D, Ariey F, Mercereau-Puijalon O. 2013. Étude de la résistance de Plasmodium falciparum aux antipaludiques au sein du réseau international des Instituts Pasteur (RIIPPalu). Med. Sci., 29(6-7): 647-655. DOI : https://doi.org/10.1051/medsci/20132960 20

Ministère de la Santé Publique Tchad. 2013. Rapport final de l'Enquête Nationale sur les Indicateurs du Paludisme au Tchad 2010 (ENIPT-2010). Rapport du Ministère de la Santé Publique Tchad.
Mukomena SE, Philipe CM, Désiré MK, Pascal LT, Ali MM, Oscar LN. 2016. Parasitémie asymptomatique chez les enfants de moins de 5 ans, enfants en âge scolaire et prise en charge des épisodes fébriles dans les ménages de Lubumbashi, République Démocratique du Congo. The Pan African Medical Journal, 24.

Organisation Mondiale de la Santé. 2015. Rapport sur le paludisme dans le monde 2014, Résume. Rapport OMS, Genève, $56 \mathrm{p}$.

Organisation Mondiale de la Santé. 2016. Rapport sur le paludisme dans le monde 2014, Résume. Rapport OMS, Genève, $44 \mathrm{p}$.

Programme National de Lutte contre le Paludisme (PNLP), INFO-STAT, ICF Macro. 2010. Enquête sur la prévalence de l'Anémie et de la Parasitémie palustre chez les enfants (EA\&P) au Mali. PNLP/ INFO-STAT/ ICF Macro.

Tatoloum A. 2016. Contribution à l'étude de la vulnérabilité sociale urbaine au risque palustre. Cas de la ville de Moundou au Tchad. Rev. Scient. Tch., 1(9): 35-47.

Yandaï FH, Moundine K, Djoumbe E, Boulotigam K, Moukenet A, Kodindo ID, Hinzoumbe CK. 2017. Perception de risques du paludisme et utilisation des moustiquaires au Tchad. Int. J. Biol. Chem. Sci., 10(6): 26468-2654. DOI: https://dx.doi.org/10.4314/ijbcs.v10i6.19 